\title{
Differential Effects of Raloxifene and Estrogen on Insulin Sensitivity in Postmenopausal Women
}

\author{
Cathy C. Lee, MD, "† Josephine Z. Kasa-Vubu, MD* and Mark A. Supiano, MD"†
}

OBJECTIVES: To test the hypothesis that both raloxifene and estrogen would improve insulin sensitivity in postmenopausal women and that the magnitude of the effect would be similar for both drugs.

DESIGN: Placebo-controlled, double-blind, randomized study.

SETTING: The General Clinical Research Center of the University of Michigan Medical Center, a university hospital.

PARTICIPANTS: Forty-four healthy postmenopausal women $73 \pm 7$ years old (mean age \pm standard deviation) who were not receiving hormone replacement therapy.

INTERVENTION: Eight weeks of drug therapy with randomization to raloxifene $(\mathrm{n}=16)$, estrogen $(\mathrm{n}=14)$, or placebo $(\mathrm{n}=14)$.

MEASUREMENTS: These subjects underwent a frequently sampled intravenous glucose tolerance test to determine insulin sensitivity $\left(\mathrm{S}_{\mathrm{I}}\right)$ and total and regional (central) body composition measurements by dual-energy $\mathrm{x}$-ray absorptiometry at baseline and after 8 weeks of drug therapy.

RESULTS: There were no statistically significant differences in age, body mass index, total or central fat mass, or $S_{I}$ between the three groups at baseline. The major outcome variable was $S_{\text {I }}$. After 8 weeks of drug therapy, there was no significant change in $S_{I}$ in the placebo group or in the estrogen group and a significant decrease in $S_{I}$ in the raloxifene group, $P=.003$.

From the "University of Michigan Health System, Ann Arbor, Michigan; and IVA Ann Arbor Healthcare Systems, Ann Arbor, Michigan.

This work was supported in part by a Hartford/American Federation for Aging Research Academic Geriatric Fellowship award, by the University of Michigan Institute of Gerontology (T32 AG00114 "Multidisciplinary Research Training in Aging”), by the General Clinical Research Center (NIH Grant RR-00042), by the Department of Veterans Affairs Ann Arbor Geriatric Research Education and Clinical Center, by the Claude D. Pepper Older Americans Center at the University of Michigan (AG 08808), and by the Michigan Diabetes Research and Training Center (NIDDK P60DK2572).

Address correspondence to Cathy C. Lee, MD, VA Ann Arbor Healthcare System, 2215 Fuller Road, GRECC 11-G, Ann Arbor, MI 48105. E-mail: clchopra@umich.edu.
CONCLUSION: In contrast to estrogen's ability to maintain insulin sensitivity, raloxifene decreases insulin sensitivity in healthy nondiabetic postmenopausal women. The clinical significance of this effect of raloxifene to impair insulin sensitivity in postmenopausal women warrants further evaluation in future studies. J Am Geriatr Soc 51:683-688, 2003.

Key words: raloxifene; insulin sensitivity; postmenopausal women

$\mathrm{P}$ ostmenopausal women may be more insulin resistant than premenopausal women because of many factors, including aging, increased total body fat and central adiposity, decreased aerobic capacity, and possibly estrogen deficiency. Many animal studies, ${ }^{1-3}$ epidemiological data, ${ }^{4,5}$ and some prospective human studies ${ }^{6-12}$ have reported the effect of estrogen on insulin sensitivity. In general, estrogen appears to increase insulin sensitivity. Many women do not receive postmenopausal estrogen replacement therapy either because of contraindications, poor compliance, or a personal decision against its use. ${ }^{13}$ Recent evidence concerning the adverse consequences of estrogen has renewed interest in alternatives to estrogen therapy. ${ }^{14}$ Alternatives to estrogen are now available for the treatment of osteoporosis. One of these alternatives is raloxifene. Raloxifene is an antiestrogen benzothiophene derivative classified as a selective estrogen receptor modulator (SERM). This classification is based on studies that demonstrate agonist effects on bone, cholesterol metabolism, and antagonist effects on uterine and breast tissue. ${ }^{15}$ There are no published data characterizing the effects of raloxifene on insulin sensitivity in nondiabetic postmenopausal women.

The objective of this placebo-controlled, doubleblind, randomized study was to test the following hypotheses. First, both raloxifene and estrogen will improve insulin sensitivity in postmenopausal women. Second, the magnitude of the improvement in insulin sensitivity will be similar for both drugs. In addition, to assess the effects of these drugs on androgenicity, levels of sex hormone-binding globulin (SHBG) and testosterone were measured. 


\section{METHODS}

\section{Subjects}

Forty-four healthy postmenopausal women (age range 60 86) who were not on hormone replacement therapy were studied. Subjects were recruited through the Human Subjects Core of the University of Michigan Geriatrics Center, the University of Michigan Women's Health Registry, and newspaper advertisement. Subjects were screened before study entry with a medical history; physical examination; and laboratory tests, including a complete blood count, thyroid stimulating hormone, routine chemistries, and an oral glucose tolerance test. Subjects were excluded from participation if they met criteria for diabetes mellitus from the oral glucose tolerance test; ${ }^{16}$ had any history of deep venous thrombosis, pulmonary embolism, uterine cancer, or breast cancer; or had contraindications to estrogen or evidence from the history, physical examination, or laboratory testing of other significant underlying medical or psychiatric illness. Each subject gave written informed consent that was approved by the University of Michigan Human Use Committee.

\section{Study Protocol}

Subjects who fulfilled eligibility criteria were randomized in a double-blind manner to receive placebo, estrogen $(0.625 \mathrm{mg} / \mathrm{d})$, or raloxifene $(60 \mathrm{mg} / \mathrm{d})$. The tablets were placed into identical-sized opaque blue capsules to ensure blinding. These doses were chosen because they are the standard ones used in clinical practice for prevention or treatment of osteoporosis in postmenopausal women.

An 8-week comparison of raloxifene, estrogen, and placebo was proposed for two reasons. First, a previous study ${ }^{12}$ suggested that insulin sensitivity determined from an insulin tolerance test was increased after 8 weeks of conjugated estrogen therapy. Second, because combination therapy with estrogen and progesterone would be complicated by the known confounding of progesterone to decrease insulin sensitivity, ${ }^{17}$ the exposure to unopposed estrogen was limited.

These subjects underwent a frequently sampled intravenous glucose tolerance test (FSIVGTT) to determine insulin sensitivity $\left(\mathrm{S}_{\mathrm{I}}\right)$, and total and regional (central) body composition measurements by dual energy x-ray absorptiometry (DEXA, Lunar model DPX-L, Madison, WI) at baseline and after 8 weeks of drug therapy.

\section{Measurement of Insulin Sensitivity}

An FSIVGTT was performed as previously described ${ }^{18}$ with the addition of insulin to enhance precision of the estimates of insulin action. ${ }^{19}$ This method has been found to yield estimates of insulin sensitivity that are reproducible $(14 \% \text { mean coefficient of intraindividual variation })^{20}$ and comparable to the glucose clamp method. ${ }^{21}$ Subjects were instructed to consume a 200 -g carbohydrate weight-maintaining diet for 3 days before the study. Subjects recorded their diet for 3 days before the study in a 3-day food diary that was reviewed to confirm their carbohydrate intake. They then reported to the Clinical Research Center after an overnight (12-hour) fast and were studied in the supine position. An intravenous catheter was placed in the antecubital vein of one arm for the injection of glucose and insulin. Another catheter was placed in a retrograde manner into a dorsal hand vein of the contralateral arm, which was then placed into a warming box heated to $60^{\circ} \mathrm{C}$ to obtain arterialized-venous blood samples for glucose and insulin. Twenty minutes after the intravenous lines were inserted, three baseline samples for glucose and insulin were obtained at 5 -minute intervals. At 0 minutes, $50 \%$ glucose $(300 \mathrm{mg} /$ $\mathrm{kg}$ ) was given as an intravenous push over 30 seconds. Blood samples were collected at 2, 3, 4, 5, 6, 8, 10, 12, 14, $16,19,22,23,24,25,27,30,40,50,60,70,80,90,100$, $120,140,160$, and 180 minutes after the glucose bolus. Insulin $(0.02 \mathrm{U} / \mathrm{kg})$ was given intravenously at 20 minutes.

\section{Measurements of Body Composition}

Lean body mass and total body composition were determined using DEXA. ${ }^{22}$ The DEXA measure of abdominal adiposity or central fat mass was determined with the manual analysis component of the Lunar software package (Lunar software version $4.5 \mathrm{c}$, extended research analysis, Madison, WI). A rectangle was drawn on the digital scan image bounded superiorly by the horizontal line identifying the T12/L1 intervertebral space, inferiorly by the horizontal line denoting the L4/L5 intervertebral space, and bilaterally by connecting the two horizontal lines in a region free of tissue. Abdominal adiposity was defined as the fat mass within this region. Total bone mineral density was also measured, although no significant change was anticipated following the short-term (8-week) treatment period.

\section{Measurement of Androgenicity}

Plasma testosterone by chemiluminescence and plasma free testosterone by radioimmunoassay (RIA) were measured in the Core Laboratory of the University of Michigan General Clinical Research Center. Plasma SHBG levels were measured using a solid-phase, two-site chemiluminescent enzyme immunometric assay for use with the Immulite Automated Analyzer (Immulite SHBG, Diagnostic Product Corp., Los Angeles, CA). Plasma samples were collected during the baseline period of the FSIVGTT and stored at $-70^{\circ} \mathrm{C}$ until assayed. All samples were analyzed in the same assay. The sensitivity of the SHBG assay was $0.2 \mathrm{nmol} / \mathrm{L}$. The intra-assay and interassay coefficients of variation were $6.5 \%$ and $8.7 \%$, respectively. The free androgen index was calculated with the formula. ${ }^{23}$

\section{$3.467 \times$ total testosterone $(\mathrm{ng} / \mathrm{dL}) / \mathrm{SHBG}(\mathrm{nmol} / \mathrm{L})$}

\section{Data and Statistical Analysis}

Blood samples for plasma glucose and insulin were collected in chilled glass tubes containing sodium heparin, stored on ice, and separated immediately after each study. Plasma was stored at $-70^{\circ} \mathrm{C}$ until assay. Plasma glucose was measured using the autoanalyzer glucose oxidase method and plasma insulin by RIA in the Core Laboratory of the Michigan Diabetes Research and Training Center. The $S_{I}$ and a measure of the fractional glucose turnover rate at the basal insulin level $\left(S_{G}\right)$ were calculated from a least squares fitting of the temporal pattern glucose and insulin throughout the FSIVGTT using the minimal model program. ${ }^{21}$ The average fractional standard deviation (FSD) for $S_{I}$ was 9.75 , with a range of 1.53 to 50.08 . The average FSD for $S_{G}$ was 16.14 , with a range of 2.53 to 
116.40. The acute insulin response to intravenous glucose $\left(\mathrm{AIR}_{\mathrm{G}}\right)$ was calculated as the mean rise in plasma insulin above baseline at 3, 4, and 5 minutes after intravenous glucose administration. $\mathrm{K}_{\mathrm{G}}$, a measure of glucose tolerance, is the rate of plasma glucose disappearance calculated as the least-squares slope of the natural logarithm of absolute glucose concentration between 10 and 20 minutes after the glucose bolus (a normal value for $\mathrm{K}_{\mathrm{G}}$ is greater than $1 \% / \mathrm{min}$ ).

Values are presented as mean \pm standard error of the mean, except for the subject characteristics and body composition measurements, which are presented as mean \pm standard deviation. The major outcome variable was $\mathrm{S}_{\mathrm{I}}$. From a previous placebo-controlled study on insulin sensitivity from a laboratory at the University of Michigan Health System, it was noted that the standard deviation of the difference in $S_{I}$ after 8 weeks in placebo group subjects was approximately 0.98 and that the mean $S_{I}$ was $3 \times$ $10^{-4} / \mathrm{min} / \mu \mathrm{U} / \mathrm{mL} .^{24}$ Given these values, calculation of a sample size to detect a treatment effect of a $30 \%$ increase in $S_{I}$ relative to placebo with $80 \%$ power at an $\alpha=.05$ yielded a sample size of 15 subjects per group.

Body mass index $\left(\mathrm{BMI}, \mathrm{kg} / \mathrm{m}^{2}\right)$ was determined by dividing subjects weight by their height squared. Central fat mass and total percentage body fat were measured using DEXA. Data were analyzed using SAS version 6.12 (SAS Institute Inc., Cary, NC) and GraphPad Prism version 3.00 for Windows (GraphPad Software, San Diego, CA). Group differences in baseline characteristics were analyzed using analysis of variance (ANOVA). The effect of drug therapy on change in $S_{I}\left(S_{I}\right.$, week 8 $-S_{I}$, baseline $)$ was also analyzed using ANOVA. Significant group differences from the ANOVA were then analyzed using Tukey multiple comparison test. In addition, to control for any differences in baseline characteristics that were not statistically significant, multiple regression models were constructed to evaluate whether any differences in baseline body composition or baseline $S_{\text {I }}$ were significant contributors to posttreatment $S_{\mathrm{I}}$. The effect of drug therapy on SHBG levels was similarly analyzed using ANOVA and Tukey multiple comparison test for any significant group differences from the ANOVA. SHBG levels could only be determined in 35 of the 44 subjects. Values of $P \leq .05$ were considered to be statistically significant.

\section{RESULTS}

\section{Subject Characteristics}

The mean age of these women was 73 , with a range of 60 to 86 . These women were somewhat overweight, as re- flected by their BMI $\left(26.4 \pm 4.7 \mathrm{~kg} / \mathrm{m}^{2}\right)$, total percentage body fat $(39.6 \pm 9.1 \%)$, and central fat mass $(2,626 \pm$ $1,293 \mathrm{~g})$. There were no statistically significant differences with respect to age or body composition between the placebo, estrogen, and raloxifene subject groups at baseline (Table 1). As expected, there were significant inverse relationships observed between $S_{I}$ and measures of obesity (BMI, $r=-0.57$; percentage body fat, $r=-0.56$; and central fat mass, $r=-0.53$; all $P<.001$ ).

\section{Effect of Drug Treatment on Body Composition Measurements}

Body composition measurements were also performed following 8 weeks of drug therapy to exclude a confounding effect of any changes in weight or body composition on insulin sensitivity. As anticipated, there were no significant changes in weight, BMI, total percentage body fat, central fat mass, or bone mineral density (data not shown) after 8 weeks of drug therapy (Table 1).

\section{Effect of Drug Treatment on FSIVGTT Measurements}

There were no statistically significant differences with respect to fasting glucose, fasting insulin, $\mathrm{S}_{\mathrm{I}}, \mathrm{S}_{\mathrm{G}}, \mathrm{K}_{\mathrm{G}}$, or $\mathrm{AIR}_{\mathrm{G}}$ between the placebo, estrogen, and raloxifene subject groups at baseline. Except for $S_{I}$ and fasting glucose, none of these values was significantly altered following 8 weeks of placebo, raloxifene, or estrogen therapy (Table 2). Fasting glucose levels were significantly decreased in the estrogen group after 8 weeks of therapy. There was a statistically significant group difference in the $\Delta \mathrm{S}_{\mathrm{I}}$ following 8 weeks of drug therapy $(P=.003)$ (Figure 1). Post hoc analysis using Tukey multiple comparison test demonstrated that only the effect of raloxifene to decrease $S_{I}$ was significantly different from the placebo and estrogen groups. There was no difference between the effects of placebo and estrogen therapy on $\Delta S_{I}$. This group difference was also statistically significant in a multiple regression model that controlled for subjects' baseline body composition and $\mathrm{S}_{\mathrm{I}}(P=.04)$.

\section{Effect of Drug Treatment on Androgenicity Measurement}

There were no statistically significant differences in SHBG levels, total testosterone levels, free testosterone levels, or the free androgen index between the three groups at baseline (Table 2). After 8 weeks of drug therapy, SHBG levels were significantly higher in the estrogen group $(\triangle \mathrm{SHBG}=$ $33.8 \pm 11.3 \mathrm{nmol} / \mathrm{L})$ than in the placebo $(\Delta \mathrm{SHBG}=1.3 \pm$ $2.3 \mathrm{nmol} / \mathrm{L})$ and raloxifene $(\Delta \mathrm{SHBG}=6.1 \pm 2.9 \mathrm{nmol} / \mathrm{L})$

Table 1. Baseline Subject Body Composition Measurements

\begin{tabular}{|c|c|c|c|c|c|c|}
\hline \multirow{3}{*}{$\begin{array}{l}\text { Body Composition } \\
\text { Measurement }\end{array}$} & \multicolumn{2}{|c|}{ 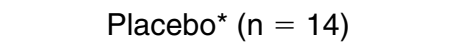 } & \multicolumn{2}{|c|}{ Estrogen $^{\dagger}(n=14)$} & \multicolumn{2}{|c|}{ Raloxifene $^{\ddagger}(n=16)$} \\
\hline & Baseline & 8 Weeks & Baseline & 8 Weeks & Baseline & 8 Weeks \\
\hline & \multicolumn{6}{|c|}{ Mean \pm Standard Deviation } \\
\hline Body mass index, $\mathrm{kg} / \mathrm{m}^{2}$ & $27.0 \pm 5.1$ & $27.1 \pm 5.1$ & $27.1 \pm 4.6$ & $27.3 \pm 4.6$ & $25.1 \pm 4.6$ & $25.1 \pm 4.6$ \\
\hline Total body fat, \% & $39.1 \pm 9.3$ & $38.5 \pm 8.7$ & $40.8 \pm 8.6$ & $41.0 \pm 8.9$ & $39.3 \pm 8.9$ & $40.2 \pm 9.2$ \\
\hline Central fat mass, $\mathrm{g}$ & $2,930 \pm 1,385$ & $2,850 \pm 1,333$ & $2,821 \pm 1,196$ & $2,583 \pm 1,199$ & $2,191 \pm 1,253$ & $2,162 \pm 1,305$ \\
\hline
\end{tabular}

Mean age \pm standard deviation: ${ }^{*} 70 \pm 8, \uparrow 75 \pm 6,{ }^{\ddagger} 73 \pm 7$. 
Table 2. Glucose Tolerance, Insulin Sensitivity, and Androgenicity Measurements

\begin{tabular}{|c|c|c|c|c|c|c|}
\hline \multirow[b]{3}{*}{ Measurement } & \multicolumn{2}{|c|}{ Placebo $(n=14)$} & \multicolumn{2}{|c|}{ Estrogen $(n=14)$} & \multicolumn{2}{|c|}{ Raloxifene $(n=16)$} \\
\hline & Baseline & 8 Weeks & Baseline & 8 Weeks & Baseline & 8 Weeks \\
\hline & \multicolumn{6}{|c|}{ Mean \pm Standard Error of the Mean } \\
\hline Fasting glucose, mg/dL & $100 \pm 3.3$ & $100 \pm 2.8$ & $106 \pm 2.5$ & $99 \pm 2.8^{*}$ & $101 \pm 2.4$ & $97 \pm 2.0$ \\
\hline Fasting insulin, $\mu \mathrm{U} / \mathrm{mL}$ & $12 \pm 1.0$ & $13 \pm 1.2$ & $13 \pm 1.7$ & $11 \pm 1.1$ & $11 \pm 1.3$ & $10 \pm 1.4$ \\
\hline$S_{G}, \min ^{-1}$ & $0.018 \pm 0.001$ & $0.016 \pm 0.002$ & $0.017 \pm 0.002$ & $0.017 \pm 0.002$ & $0.015 \pm 0.002$ & $0.020 \pm 0.002$ \\
\hline $\mathrm{K}_{\mathrm{G}}, \% / \mathrm{min}$ & $1.6 \pm 0.2$ & $1.7 \pm 0.3$ & $1.6 \pm 0.2$ & $1.2 \pm 0.2$ & $1.6 \pm 0.1$ & $1.7 \pm 0.2$ \\
\hline $\mathrm{AIR}_{\mathrm{G}}, \mathrm{pM}$ & $378 \pm 63$ & $351 \pm 47$ & $365 \pm 69$ & $326 \pm 66$ & $384 \pm 62$ & $415 \pm 66$ \\
\hline $\mathrm{S}_{\mathrm{l}}\left(\times 10^{-4} / \mathrm{min} / \mu \mathrm{U} / \mathrm{ml}\right)$ & $3.2 \pm 0.5$ & $3.3 \pm 0.5$ & $2.6 \pm 0.3$ & $3.1 \pm 0.4$ & $3.9 \pm 0.5$ & $3.1 \pm 0.5^{\star}$ \\
\hline Total testosterone, $\mathrm{ng} / \mathrm{mL}$ & $0.16 \pm 0.02$ & $0.12 \pm 0.02$ & $0.16 \pm 0.02$ & $0.12 \pm 0.02$ & $0.15 \pm 0.02$ & $0.14 \pm 0.02$ \\
\hline Free testosterone, $\mathrm{pg} / \mathrm{mL}$ & $0.61 \pm 0.10$ & $0.64 \pm 0.09$ & $0.67 \pm 0.12$ & $0.49 \pm 0.06$ & $0.50 \pm 0.08$ & $0.56 \pm 0.07$ \\
\hline $\mathrm{SHBG}, \mathrm{nmol} / \mathrm{L}^{\dagger}$ & $44.9 \pm 7$ & $46.5 \pm 6$ & $56.0 \pm 10$ & $103.3 \pm 14^{\star}$ & $58.1 \pm 7$ & $65.6 \pm 7$ \\
\hline Free androgen index ${ }^{\dagger}$ & $1.64 \pm 0.42$ & $1.17 \pm 0.34$ & $1.39 \pm 0.30$ & $0.54 \pm 0.14^{*}$ & $1.09 \pm 0.19$ & $0.81 \pm 0.17$ \\
\hline
\end{tabular}

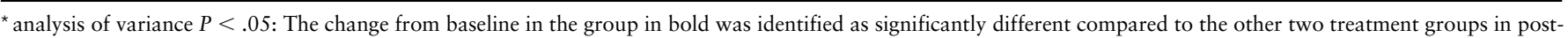
hoc analysis.

${ }^{\dagger} \mathrm{n}=35$.

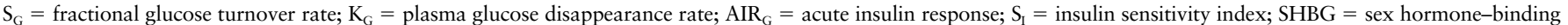
globulin.

groups, $P=.003$. Estrogen also significantly decreased the free androgen index, whereas placebo and raloxifene did not $(P=.02)$ (Table 2). There was a trend for estrogen to decrease the free testosterone levels after 8 weeks of therapy, but this was not statistically different compared with the placebo and raloxifene groups.

\section{DISCUSSION}

Contrary to the initial hypotheses, insulin sensitivity was maintained in the estrogen group, whereas raloxifene therapy was associated with a significant decrease in insulin sensitivity in healthy nondiabetic postmenopausal women. Raloxifene therapy shares some of estrogen's properties, such as the ability to improve bone density and the cholesterol profile. ${ }^{25,26}$ Postmenopausal estrogen replacement therapy has been reported to increase insulin sensitivity in some studies. ${ }^{1,4,6}$ Because raloxifene shares some effects of estrogen therapy, it was hypothesized that it would like-

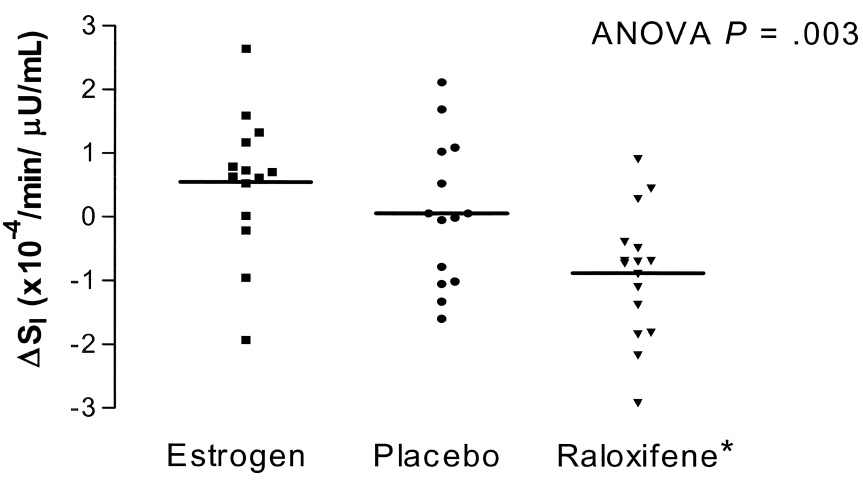

Figure 1. The change in insulin sensitivity $\left(\Delta \mathrm{S}_{\mathrm{I}}\right)$ following 8 weeks of drug therapy with estrogen, placebo, or raloxifene in healthy nondiabetic postmenopausal women. $\mathrm{S}_{\mathrm{I}}$ was significantly lower in the raloxifene group* than in the estrogen and placebo groups (analysis of variance (ANOVA) $P=.003$ ). wise increase insulin sensitivity in nondiabetic postmenopausal women, but in the current study, after 8 weeks of therapy with raloxifene, insulin sensitivity was significantly decreased.

No prior studies have investigated whether there is an effect of raloxifene on insulin sensitivity in nondiabetic postmenopausal women. One study conducted in postmenopausal women with type 2 diabetes mellitus found no significant effect of 3 months of raloxifene therapy on glucose disposal rates, ${ }^{27}$ but, given the degree of insulin resistance in this diabetic study population, it would be difficult to detect a further decline in insulin sensitivity as observed in the nondiabetic subjects in the current study.

The decrease in insulin sensitivity seen in the raloxifene group was not associated with an increase in BMI, central fat mass, or percentage body fat. This is consistent with other longer-duration studies that have shown no change in BMI with raloxifene. ${ }^{28}$ This suggests that the decrease in insulin sensitivity with raloxifene seen in this study may involve other mechanisms besides changes in body composition.

In the current study, one of the differences noted in the actions of raloxifene and estrogen was their effect on SHBG levels and the free androgen index. SHBG has been used as an indirect measure of androgenicity; estrogen therapy has been shown to increase SHBG and testosterone therapy to decrease SHBG levels..$^{29}$ As expected, in this study, estrogen significantly increased SHBG levels and decreased the free androgen index, suggesting a decrease in androgenicity. In contrast, raloxifene did not alter SHBG levels or the free androgen index. There may be an association between androgenicity and insulin sensitivity. There is evidence that an increase in androgenicity may contribute to the development of insulin resistance in postmenopausal women. In two prospective studies, low SHBG levels (greater androgenicity) have been linked to the development of noninsulin-dependent diabetes mellitus in women. ${ }^{30,31} \mathrm{~A}$ previous study showed that low SHBG 
levels in postmenopausal women were significantly associated with decreased insulin sensitivity (unpublished data). There is also evidence that hyperinsulinemia contributes to increased androgenicity. ${ }^{32-34}$

Raloxifene and estrogen also have differences at the molecular level that may contribute to the different effects that were observed on insulin sensitivity and androgenicity. Studies have shown that there is more than one type of estrogen receptor, estrogen receptor $\alpha(\mathrm{ER} \alpha)$ and estrogen receptor $\beta$ (ER $\beta) .{ }^{35}$ These two types of estrogen receptors are found in different tissue distributions. Estrogen is primarily an ER $\alpha$ agonist, whereas raloxifene is a partial $\mathrm{ER} \alpha$ agonist and a potent ER $\beta$ agonist. ${ }^{36}$ Differential tissue responses may also be due to the interactions of estrogen and raloxifene with one of several deoxyribonucleic acid response elements that interact with estrogen receptors. $^{37,38}$ In addition, different tissues may have selective responses to estrogens than to SERMs. ${ }^{39}$ These clinical and molecular differences may account for different effects of raloxifene and estrogen on insulin sensitivity.

This study has several potential limitations. It tested a single dose of estrogen and raloxifene for a short-term, 8 -week, duration. It is unclear what the dose equivalency is between raloxifene and estrogen. These doses were chosen because they are the standard ones used in clinical practice for prevention or treatment of osteoporosis in postmenopausal women. Because the study was terminated after 8 weeks of treatment, the effect that longer use of raloxifene or estrogen would have on insulin sensitivity or androgenicity cannot be predicted. Also, because only the effect of unopposed estrogen was studied, the results cannot be extended to the more-common clinical use of estrogen in combination with progesterone therapy. This study was not designed to infer any effect of estrogen or raloxifene on cardiovascular risk, nor was it designed to assess an effect on bone density. Further studies would be helpful to confirm these results and clarify questions about dosing, duration, and long-term effects of these therapies.

In summary, after 8 weeks of drug therapy, in contrast to the lack of an effect of estrogen on insulin sensitivity, raloxifene decreased insulin sensitivity in healthy nondiabetic postmenopausal women. The clinical significance of this effect of raloxifene of impairing insulin sensitivity in nondiabetic postmenopausal women warrants further evaluation in future studies.

\section{ACKNOWLEDGMENTS}

We thank Cathie Bloem, RN, for her assistance with subject recruitment, subject care, follow-up, and technical assistance; Marla Smith for her technical assistance; Kathy Jarvenpa, RN, and the staff of the General Clinical Research Center for their care of our subjects during this study; and Andrzej Galecki, MD, PhD, for his statistical advice. Eli Lilly and Company donated raloxifene.

\section{REFERENCES}

1. McKerns KW, Coulomb B, Kaleita E et al. Some effects of in vivo administered estrogens on glucose metabolism and adrenal cortical secretion in vitro. Endocrinology 1958;63:709-722.

2. Costrini NV, Kalkhoff RK. Relative effects of pregnancy, estradiol, and progesterone on plasma insulin and pancreatic islet insulin secretion. J Clin Invest 1971:50:992-999.
3. Bailey CJ, Ahmed-Sorour H. Role of ovarian hormones in the long-term control of glucose homeostasis. Diabetologia 1980;19:475-481.

4. Barrett-Connor E, Laakso M. Ischemic heart disease risk in postmenopausal women. Effects of estrogen use on glucose and insulin levels. Arteriosclerosis 1990;10:531-534.

5. Hammond CB, Jelovsek FR, Lee KL et al. Effects of long-term estrogen replacement therapy. Am J Obstet Gynecol 1979;133:525-536.

6. Notelovitz M, Johnston M, Smith S et al. Metabolic and hormonal effects of 25 -mg and 50-mg 17 $\beta$-estradiol implants in surgically menopausal women. Obstet Gynecol 1987;70:749-754.

7. Spellacy WN, Buhi WC, Birk SA. The effect of estrogens on carbohydrate metabolism: Glucose, insulin, and growth hormone studies on one hundred and seventy-one women ingesting Premarin, mestranol, and ethinyl estradiol for six months. Am J Obstet Gynecol 1972;114:378-392.

8. Cagnacci A, Soldani R, Carriero PL et al. Effects of low doses of transdermal $17 \beta$-estradiol on carbohydrate metabolism in postmenopausal women. J Clin Endocrinol Metab 1992;74:1396-1400.

9. Ajabor LN, Tsai CC, Vela P et al. Effect of exogenous estrogen on carbohydrate metabolism in postmenopausal women. Am J Obstet Gynecol 1972; 113:383-387.

10. Godsland IF, Gangar K, Walton C et al. Insulin resistance, secretion, and elimination in postmenopausal women receiving oral or transdermal hormone replacement therapy. Metabolism 1993;42:846-853.

11. Lindheim SR, Presser SC, Ditkoff EC et al. A possible bimodal effect of estrogen on insulin sensitivity in postmenopausal women and the attenuating effect of added progestin. Fertil Steril 1993;60:664-667.

12. Lindheim SR, Buchanan TA, Duffy DM et al. Comparison of estimates of insulin sensitivity in pre- and postmenopausal women using the insulin tolerance test and the frequently sampled intravenous glucose tolerance test. J Soc Gynecol Invest 1994;1:150-154.

13. Willhite SL, Goebel SR, Scoggin JA. Raloxifene provides an alternative for osteoporosis prevention. Ann Pharmacother 1998;32:834-837.

14. Hulley S, Grady D, Bush T et al. Randomized trial of estrogen plus progestin for secondary prevention of coronary heart disease in postmenopausal women. JAMA 1998;280:605-613.

15. Kauffman RF, Bensch WR, Roudebush RE et al. Hypocholesterolemic activity of raloxifene (LY139481): Pharmacological characterization as a selective estrogen receptor modulator. J Pharmacol Exp Ther 1997;280:146-153.

16. The Expert Committee on the Diagnosis and Classification of Diabetes Mellitus. Report of the expert committee on the diagnosis and classification of diabetes mellitus. Diabetes Care 1998;21(Suppl. 1):S5-S19.

17. Lindheim SR, Presser SC, Ditkoff EC et al. A possible bimodal effect of estrogen in insulin sensitivity in postmenopausal women and the attenuating effect of added progestin. Menopause 1993;60:664-667.

18. Bergman R. Toward physiological understanding of glucose tolerance: Minimal model approach. Diabetes 1989;38:1512-1527.

19. Yang Y, Youn J, Bergman R. Modified protocols improve insulin sensitivity estimation using the minimal model. Am J Physiol 1987;253:E595-E602.

20. Ferrari P, Alleman Y, Shaw S et al. Reproducibility of insulin sensitivity by the minimal model method. Diabetologia 1991;34:527-530.

21. Bergman R, Prager R, Volund A et al. Equivalence of the insulin sensitivity index in man derived by the minimal model method and the euglycemic glucose clamp. J Clin Invest 1987;79:790-800.

22. Mazess R, Barden H, Bisek J et al. Dual-energy x-ray absorptiometry for total-body and regional bone-mineral and soft-tissue composition. Am J Clin Nutr 1990;51:1106-1112.

23. Wilke TJ, Utley DJ. Total testosterone, free-androgen index, calculated free testosterone, and free testosterone by analog RIA compared in hirsute women and in otherwise-normal women with altered sex-hormone-bindingglobulin. Clin Chem 1987;33:1372-1375.

24. Supiano MA, Hogikyan RV, Morrow LA et al. Hypertension and insulin resistance: Role of sympathetic nervous system activity. Am J Physiol 1992; 263:E935-E942.

25. Ettinger B, Black D, Mitlak BH et al. Reduction of vertebral fracture risk in a postmenopausal women with osteoporosis treated with raloxifene: Results from a 3-year randomized clinical trial. JAMA 1999;282:637-645.

26. Delmas PD, Bjarnason NH, Mitlak BH et al. Effects of raloxifene on bone mineral density, serum cholesterol concentrations, and uterine endometrium in postmenopausal women. N Engl J Med 1997;337:1641-1647.

27. Andersson B, Johannsson G, Holm G et al. Raloxifene does not affect insulin sensitivity or glycemic control in postmenopausal women with type 2 diabetes mellitus: A randomized clinical trial. J Clin Endocrinol Metab 2002;87: 122-128.

28. Oleksik AM, Duong T, Pliester N et al. Effects of the selective estrogen receptor modulator raloxifene on the somatotropic axis and insulin-glucose homeostasis. J Clin Endocrinol Metab 2001;86:2763-2768.

29. Anderson DC. Sex-hormone-binding globulin. Clin Endocrinol 1974;3:69-96. 30. Haffner SM, Valdez RA, Morales PA et al. Decreased sex hormone-binding 
globulin predicts noninsulin-dependent diabetes mellitus in women but not in men. J Clin Endocrinol Metab 1993;77:56-60.

31. Linstedt G, Lundberg P-A, Lapidus L et al. Low sex-hormone-binding globulin concentration as independent risk factor for development of NIDDM, 12yr follow-up of population study of women in Gothenburg, Sweden. Diabetes 1991;40:123-128.

32. Nestler JE, Powers LP, Matt DW et al. A direct effect of hyperinsulinemia on serum sex hormone-binding globulin levels in obese women with the polycystic ovary syndrome. J Clin Endocrinol Metab 1991;72:83-89.

33. Plymate SR, Matej LA, Jones RE et al. Inhibition of sex hormone-binding globulin production in the human hepatoma (Hep G2) cell line by insulin and prolactin. J Clin Endocrinol Metab 1988;67:460-464.

34. Nestler JE, Clore JN, Strauss JF III et al. The effects of hyperinsulinemia on serum testosterone, progesterone, dehydroepiandrosterone sulfate, and cortisol levels in normal women and in a woman with hyperandrogenism, in- sulin resistance, and acanthosis nigricans. J Clin Endocrinol Metab 1987; 64:180-184.

35. Barkhem T, Carlsson B, Nilsson Y et al. Differential response of estrogen receptor $\alpha$ and estrogen receptor $\beta$ to partial agonists/antagonists. Mol Pharmacol 1998;54:105-112.

36. Paech K, Webb P, Kuiper GGJM et al. Differential ligand activation of estrogen receptors ERalpha and ERbeta and AP1 sites. Science 1997;277:1508-1510.

37. Yang NN, Venugopalan M, Hardikar S et al. Identification of an estrogen response element activated by metabolites of $17 \beta$-estradiol and raloxifene. Science 1996;273:1222-1225.

38. Grese TA, Sluka JP, Bryant HU et al. Molecular determinants of tissue selectivity in estrogen receptor modulators. Proc Natl Acad Sci U S A 1997;94: 14105-14110.

39. Khovidhunkit W, Shoback D. Clinical effects of raloxifene hydrochloride in women. Ann Intern Med 1999;130:431-439. 